\title{
AUTOMATIC GENERATION OF COBIE DATA FROM REVIT
}

*J. Lee, W. Jeong, V. Faghihi, and J. Kang

Texas A\&M University

3137 TAMU

Langford Building A, Room 424

College Station, Texas 77843-3137

United States

(*Corresponding author: jeffyi@tamu.edu) 


\title{
AUTOMATIC GENERATION OF COBIE DATA FROM REVIT
}

\begin{abstract}
As the usage of BIM increased in the construction industry, some construction owners started exploring the use of BIM for maintaining their facilities. Construction Operations Building Information Exchange (COBIE), a spreadsheet data format suggested in 2007 to manipulate building information, is considered as one of the solutions that would facilitate the process of transferring information from BIM to applications for facilities management. Several BIM and Computer Aided Facilities Management (CAFM) applications offer functions to export or import the COBIE data. To facilitate the process of generating the COBIE data out of Building Information Model, some add-in applications, such as COBIE Toolkit for Revit, also were developed. However, most of these applications handle a limited amount of COBIE data. Professionals using these add-in applications therefore sometimes end up spending a significant amount of time to add additional data manually to the COBIE data file. To automatically get a data-rich COBIE file, we developed a prototype application that extracts information from Revit, which is one of the BIM authoring tools, stores them in an external database, and then produces the COBIE file using information stored in the database. We applied our application to a pilot project and produced 8 COBIE worksheets automatically, which demonstrated a significant amount of time saving. This paper presents how our prototype application produces 8 COBIE data sets automatically. It also addresses our attempt to handle more COBIE data sets.
\end{abstract}

\section{KEYWORDS}

BIM, COBIE, automatic generation, Revit, Facilities Management

\section{INTRODUCTION}

As Building Information Modeling (BIM) has been actively used for productivity improvement in design and construction (D\&C) (Sacks, 2004; Lee et al., 2008), many construction professionals and building owners begin to be serious about using information generated in the course of $\mathrm{D} \& \mathrm{C}$ for operating and maintaining the facilities. To better maintain facilities, professionals already have been using Computer Aided Facilities Management (CAFM) solutions, which is basically helping them make proactive decisions using information stored in the database system. Knowing that both BIM and CAFM handle building information, logical speculation would be whether we could use information stored in BIM for CAFM system, so that we do not have to punch the same information in the CAFM system again.

Construction Operations Building Information Exchange (COBIE), a spreadsheet data format suggested in 2007 (East, 2007) to manipulate building information, has been considered as one of the solutions that would facilitate the process of transferring information from BIM systems to CAFM systems. Several BIM and CAFM systems in fact offer functions to export/import information to/from the COBIE data. To facilitate the process of generating the COBIE data out of Building Information Model, some addin applications, such as COBIE Toolkit for Revit, also have been developed.

Most add-in applications developed for BIM solutions, however, handle a limited amount of COBIE data. For instance, COBIE Toolkit for Revit produces only 5 COBIE data sets out of 12 commonly used and 16 entire COBIE data sets (Autodesk, 2013). Therefore, professionals using these add-in applications sometimes end up spending a significant amount of time in order to add additional data manually to the COBIE data file. Furthermore, it is a manual process. End users have 11 stages to finally produce COBIE files from the Revit BIM model. 
We, therefore, investigate how to produce more a data-rich COBIE file from design tools automatically. We develop a prototype application and also address effort to handle more COBIE data sets from Revit BIM models.

\section{OVERVIEW OF COBIE}

COBIE has been considered as one of the solutions that would facilitate the process of transferring information from BIM systems to CAFM systems. Its purpose is improving the way to capture information in D\&C phases, and providing for operation and management phase. (East, 2007; 2012) The merit of COBIE is that it can be viewed not only in several BIM applications but also in simple spreadsheets. "This versatility enables COBIE to be used regardless of size and technological sophistication." (East, 2013)

COBIE file contains data from the entire life cycle of buildings or facilities. While there were 29 individual worksheets in every COBIE file the very first time (Table. 1), 16 worksheets are used in these days and 12 worksheets are commonly used in real projects (Table. 2). There are four, three, three, and six worksheets for design, construction, O\&M, and common area, respectively. Each worksheet includes a variety of columns which have more specific data. For instance, contract worksheet is included in contract set. Contract worksheet includes specific data like 'Email', 'CreatedBy', 'CreatedOn', 'Category', 'Company', 'Phone', 'ExternalSystem', 'Department', and so on.

Table $1-29$ Worksheets of COBIE

\begin{tabular}{ll}
\hline Set & 29 Worksheets \\
\hline Contract & Contract \\
Design & Facility, Floor, Space, System, Register, Component, Attribute, and Coordinate \\
Submittal & Schedule, Document, Transmittal, and Action \\
Installation & Installation, Manual, Warranty, and Spare \\
Commissioning & Instruction, Test, and Certification \\
Resource & Material, Tool, and Training \\
Job plan & PM, Safety, Trouble, Start-up, Shut-Down, and Emergency \\
\hline
\end{tabular}

Table $2-16$ Worksheets of COBIE

\begin{tabular}{ll}
\hline Set & 16 Worksheets \\
\hline Design & Facility, Floor, Space, Zone \\
Construction & Type, Component, System \\
O\&M & Job, Resource, Spare \\
Common & Contact, Document, Issue, Coordinate, Attribute, Connection \\
\hline
\end{tabular}

The specification of COBIE provides neutral and open standard format. It is delivered in the Spreadsheet XML 2003 format at each stage of building's life-cycle. Some of worksheets are required fields, while others are optional. Likewise, some columns in a worksheet are fundamental, while others are optional. Commercial BIM applications showed two approaches to create COBIE files. Some of applications like Autodesk Revit and Bently Architecture use IFC as a media to transfer data. The other applications like ARCHIBUS, Inc ARCHIBUS and AssetWORKS Aim produce directly from the application (East, 2013).

\section{RELATED BACKGROUND RESEARCH}

Many construction professionals have begun to study information handling for operating and maintaining phases of facilities (Lee, 2007; Yi et al., 2008; Sabol, 2008; Riddell, 2013). For the better maintenance and management of facilities, Motamedi and Hammad (2009) suggested the use of RFID (Radio frequency identification) with BIM concept. They showed a technical way to utilized BIM into the practical projects. Kasprzak and Dubler (2012), Lucas (2012), and Becerik-Gerber et al. (2012) studied BIM frameworks or BIM data handling to support facility management (FM). Lucas applied BIM 
framework particularly to the FM of health care facility. While many researchers have shown the effort to struggle with BIM and FM, COBIE is out of research scopes. Some researchers tend to consider COBIE just a medium to transfer data from D\&C phases to O\&M phase. While COBIE is not a complete implement to deliver data at each stage, researchers have studied on how to apply COBIE itself to a real project (Anderson et al., 2012).

\section{STATE-OF-THE-ART OF REVIT COBIE TOOLKIT}

While Revit is one of the most popular BIM design tools in North America, it also supports API (Application Programming Interface) to expand its potential towards the other life cycle phases of buildings or facilities. Revit COBIE Toolkit is a good sample of it. This toolkit expands its ability into FM area by producing COBIE data file from the BIM design application.

However, according to Autodesk (2013), the add-in application does not generate the COBIE file fully and automatically, and it just assists end users to produce COBIE deliverables in conjunction with their Revit model. In terms of data, it just produce 5 data sheets out of 16 sheets; ' 04 - Space', '05 - Zone', '06 - Type', '07 - Component', and '08 - System'. The data in these sheets contains design information, thus it is quite possible to be produced from BIM design application directly. While 3 other sheets such as ' 01 - Contact', '02 - Facility', and '03 - Floor' also contain design information, Revit COBIE Toolkit is not able to produce them. Furthermore, professionals using these add-in applications sometimes end up spending a significant amount of time in order to add additional data manually like ' 09 - Spare', ' 10 Resource', '11- Job', '12 - Document', '13 - Attribute', '14 - Coordinate', '15 - Connection', and '16 Issue' like Figure 1. Thus, it is required to advance the technology.

COBIE Toolkit is not an automated API. To produce a COBIE file, we needed to proceed with 11 steps. First of all, add-in is set up in the Revit project and is deployed by loading COBIE template. Then, users make systems and zones schedules. Next, it includes the families and types to be connected into the COBIE file through the schedule view of COBie2-ComponentsToSchedule. It shows additional properties of family in the property window, which will be used in the COBIE file. After inputting all data into that window, COBIE project level parameter field is populated by running an add-in application within Revit. Eventually, exports and then imports schedule data into Microsoft Excel and copies and pastes all data into the COBIE template worksheet one by one.

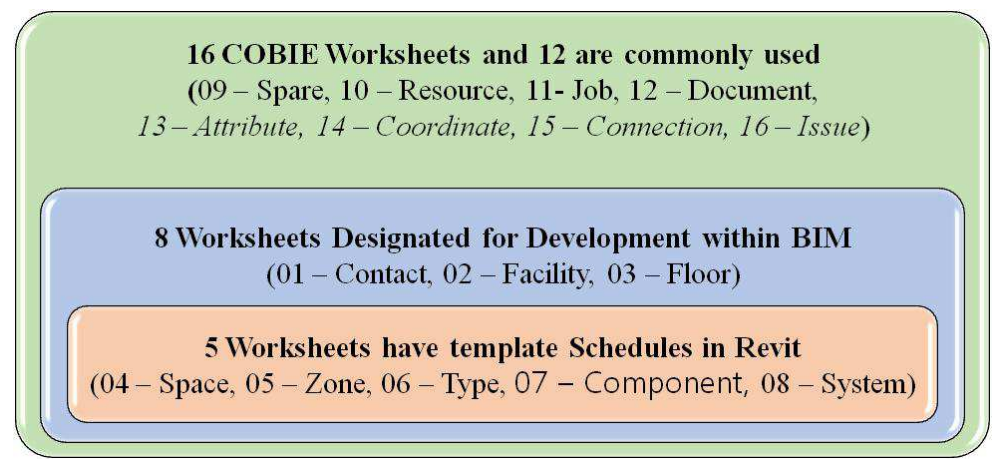

Figure 1 - COBIE spreadsheet; only 5 worksheets can be extracted from Revit project

\section{METHODOLOGY}

To produce a COBIE file, we need to export BIM model to external database, and then generate a COBIE file from DB like Figure 2. For the first step, we used 'export to ODBC' function to produce a DB for a project. Figure 3 shows the brief data structure of Revit. Before exporting the entire data, we set up the essential requirement to export to ODBC (Open DataBase Connectivity). Next, it is exported into a Microsoft SQL server. The reason for using DB is that "dynamic information repository (e.g., a DB management system) can store and return any information item generated or used in a process" (Lee et al. 
2007). In the whole life cycle of facilities, huge amount of data should be stored and used very frequently. So, it is essential to utilize the merit of DB system. Additionally, it is easier both to remove irregularities and redundancies in a data model and to define a well-organized data structure.

In the second step, we generate a COBIE file from external DB by using a module which connects DB into COBIE. This module needs a mapping relationship between DB and COBIE data structure. We figure out what kind of tables and columns are embedded in Revit through a prototype BIM model. Table 3, for instance, shows the relationship between worksheet \#2 'CONTACT' in COBIE, which was not able to be produced by using Revit Toolkit, and DB. Most of the columns are filled with the data from DB, while some of them come from other worksheets. At the first column of 'COATACT' worksheet, there is 'Email' which is a primary key. It is possible to get data from 'ProjectAddress' column of ProjectInformation' table. It is not exactly the same, but we can match similar things to transfer data. It means that we have to input email address of architects or engineers into ProjectAddress field. Likewise, we match each data field like Table 3.

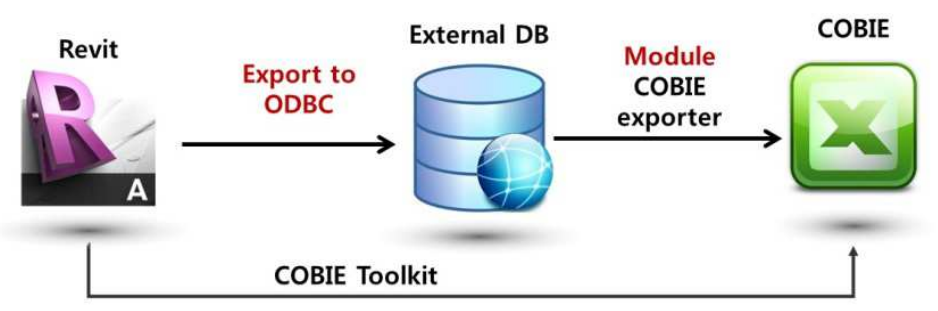

Figure 2 - Flow of data from Revit model to COBIE

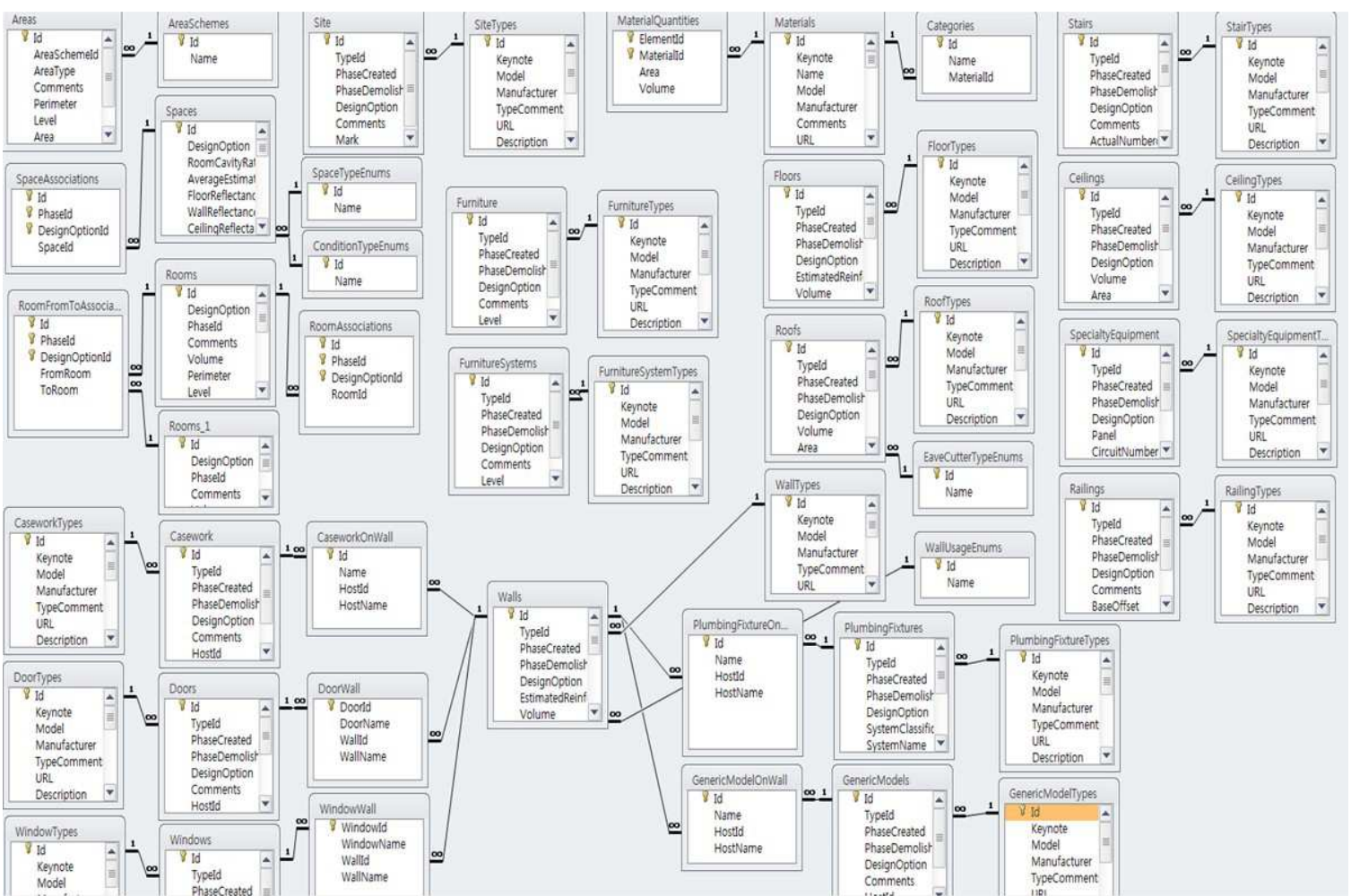

Figure 3 - DB structure of Revit 
Table 3 -Mapping Relationship between External DB and COBIE data structure

\begin{tabular}{ll|l}
\hline \multicolumn{2}{c|}{ COBIE } & \multicolumn{1}{c}{ Database } \\
\hline Col A & Email & Primary Key: ProjectInformation - ProjectAddress \\
\hline Col B & CreatedBy & *Contact - Email \\
\hline Col C $\sim$ D & CreatedOn & ProjectInformation - ClientName \\
& Category & *PickList - Category-Role \\
& Company & ProjectInformation - ProjectName \\
& Phone & ProjectInformation - ProjectNumber \\
\hline Col E $\sim$ G & ExtSystem & Name of SW. Fixed (Ex. Revit2013) \\
& ExtObject & *PickList - objContact \\
& ExtIdentifier & ProejctInformation - ID \\
\hline Col J $~ S$ & Department & Profile - TypeName \\
& OrganizationCode & New field \\
& GivenName & New field \\
& FamilyName & Profile - FamilyName \\
& Street & ProjectInformation - \\
& PostalBox & ProjectInformation - \\
& Town & New field \\
& StateRegion & New field \\
& PostalCode & New field \\
& Country & New field \\
\hline
\end{tabular}

Now, based on the mapping relationship, we use the following query command to export data from SQL 2008 server to MS Excel. We use COBIE template file which has only the titles of each column and other data fields are empty. In this command, we locate the COBIE template file in C root folder. Following commands query data of ProjectAddress column in ProjectInformation table and transfer into Email column of CONTACT worksheet in COBIE template file.

\author{
insert into OPENROWSET('Microsoft.Jet.OLEDB.4.0', \\ 'Excel 8.0;Database=C:ICOBIE_Template.xls;', \\ 'SELECT Email FROM Contact') select ProjectAddress from ProjectInformation
}

ExtSystem is unique data field and it contains the name of system. In this study, it should be 'Autodesk Revit' through the entire COBIE file. It is unique column and the value is not changeable. Thus, we input it as an invariable value. Likewise, we suggest mapping relationship for other COBIE worksheets: '01 - Contact', '02 - Facility', '03 - Floor', '04 - Space', '05 - Zone', '06 - Type', '07 - Component', and '08 - System'. With this mapping relationship, we generate COBIE file through the developed module from external DB.

\title{
DISCUSSION
}

Figure 4 shows an example of COBIE file produced from this study. It contains the information of 'Contract' to 'Space' worksheets which is not included in COBIE file created by Revit COBIE toolkit. By automatically producing COBIE file which contains more data than Revit COBIE toolkit product, we are able to reduce the total time to generate the final version of COBIE file. In this study, our object building consists of 2 stories and 4 offices including 14 spaces, 68 zones, 20 types, 50 components, and 50 systems. If we consider it takes the same time to input data into 'Common' set of COBIE worksheets in both ways, we can conclude that we save time as much as we input data into '01 - Contact', '02 - Facility', and '03 Floor'. In this study, it is about 2.5 hours because we have to check the primary key, CreatedBy', at each worksheet as well as compare with other data field. In real projects, the more complex the building is, the more we can save time. 
Additionally, transferring data directly from BIM modeling tool, it is not required to repeat and spending time by inputting the same D\&C data at O\&M phases. It enables both to reduce redundancy and reduce the chance to loss data.

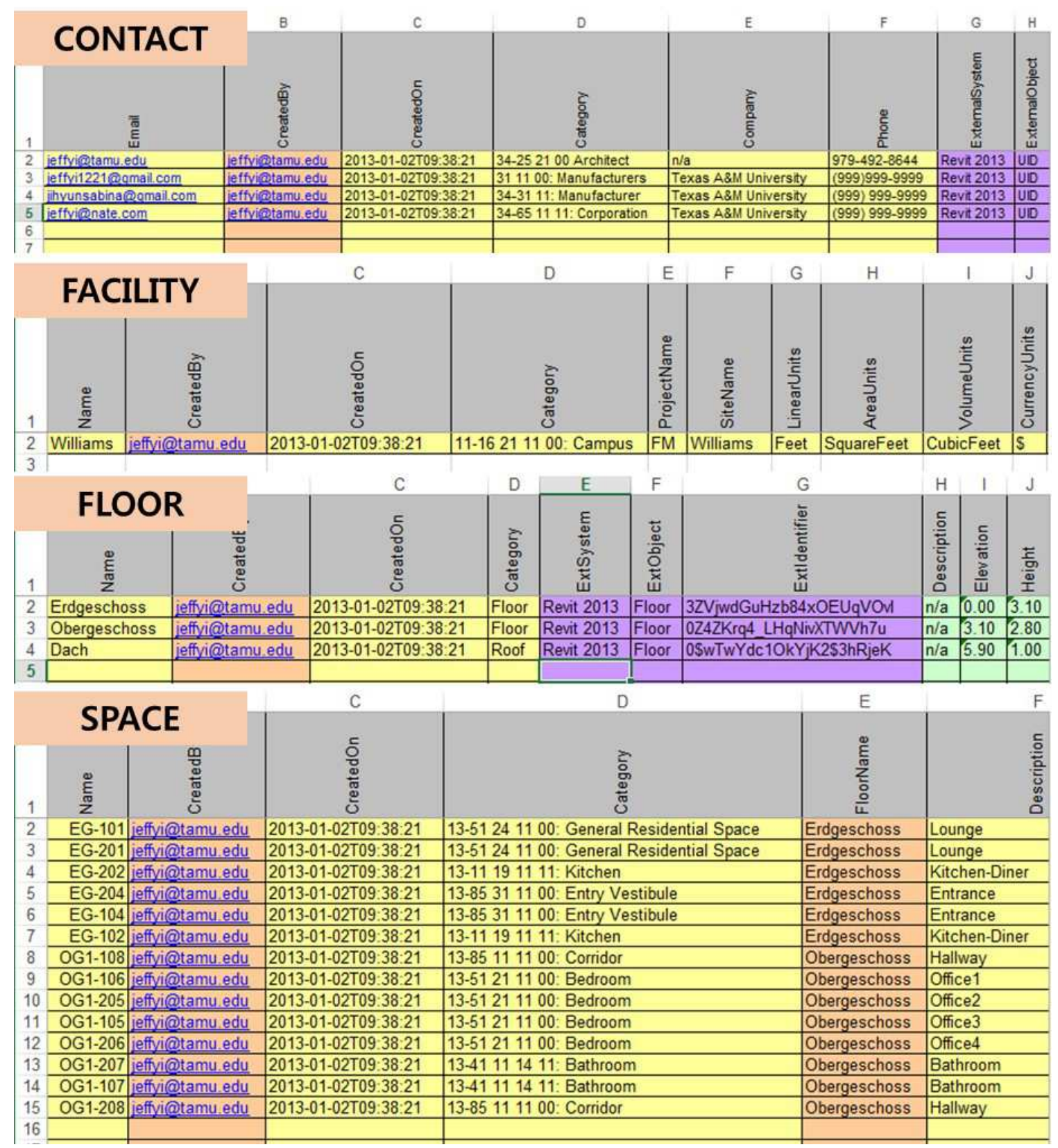

Figure 4 - Example of COBIE file

\section{CONCLUSION}

In order to get more information transferred from BIM solutions to CAFM systems automatically, we developed a prototype application that extracts information from Revit, which is one of BIM authoring tools, stores extracted information into an external DB, and then produces the COBIE data file using information stored in the DB. We applied our application to prototype projects and demonstrated that it 
produced 8 COBIE data sets automatically, which helped facilities managers save a significant amount of time.

Utilization of these modules reduced the time required to produce a COBIE file from design information. It also increased interoperability by directly delivering data from the design tool - Autodesk Revit in this research - and decreased the loss of information at each phase of the building project. It is expected that 12 data sheets of COBIE can be produced through additional investigation. Furthermore, using a private external DB has the advantage of vendor independency because software versions of BIM are no longer an issue.

\section{REFERENCES}

Anne Anderson, Andrew Marsters, Carrie Sturts Dossick, and Gina Neff (2012) "Construction to Operations Exchange: Challenges of Implementing COBie and BIM in a Large Owner Organization", Construction Research Congress 2012, 688-697.

Ali Motamedi and Amin Hammad (2009). "RFID-Assisted Lifecycle Management of Building Components Using BIM Data", 26th International Symposium on Automation and Robotics in Construction (ISARC 2009), 109-116.

Autodesk (2013). "Using the COBie Add-in Demonstration Application for Autodesk Revit 2013", Autodesk.

Bill East (2013). "Construction Operations Building Information Exchange (COBIE)", Retrieved from buildingSMART website: http://www.buildingsmartalliance.org/index.php/projects/activeprojects/25

Burcin Becerik-Gerber, Farrokh Jazizadeh, Nan Li, and Gulben Calis (2012) "Application Areas and Data Requirements for BIM-Enabled Facilities Management", Journal of Construction Engineering and Management, 138 (3), 431-442.

Christopher Riddell (2013). "BIM: Facility Management Tool of the Future", Retrieved from website: http://christopherriddell.com/Riddell_BIM_FM.pdf

Colleen Kasprzak and Craig Dubler (2012) 'Aligning BIM with FM: streamlining the process for future projects', Australasian Journal of Construction Economics and Building, 12 (4) 68-77.

Ghang Lee, Rafael Sacks, Charles Eastman (2007). "Product data modeling using GTPPM - A case study", Automation in Construction, 16, 392-407.

Jason Lucas (2012). An Integrated BIM Framework to Support Facility Management in Healthcare Environments (Doctor's dissertation). Virginia Polytechnic Institute and State University, Virginia, US.

JinHoon Lee, Ji-Hoon Lee, Hyo-Jin Kim, Sang-Ho Lee (2008). "An Extended Data Model based on the IFC for Representing Detailed Design Information of Steel Bridge Members", Journal of Computational Structural Engineering Institute of Korea, 21 (2), 253-263. (ISSN: 1225-1569)

Jin-Hoon Yi, HyunJung An, Hyo-Jin Kim \& Sang-Ho Lee (2008). "Interoperable Information Model based on IFC for the Cable-Stayed Bridge Monitoring System", Proceedings of the Fourth International IABMAS Conference, 352, Taylor \& Francis 2008, (ISBN: 978-0-415-46844-2)

Louise Sabol (2008). "Building Information Modeling \& Facility Management", IFMA World Workplace.

Rafael Sacks, Charles Eastman, and Ghang Lee (2004). "Parametric 3D modeling in building construction with examples from precast concrete". Automation in Construction, 13 (3), 291-312.

William East (2007). "Construction Operations Building Information Exchange (COBIE): Requirements Definition and Pilot Implementation Standard", US Army Corps of Engineering. Retrieved from http://www.dtic.mil/cgi-bin/GetTRDoc?AD=ADA491932\%26amp;Lo

William East (2012). "Construction Operation Building Information Exchange". USACE ERDC. Retrieved from Whole Building Design Guide: http://www.wbdg.org/resources/cobie.php

William East (2013) "Construction Operations Building Information Exchange (COBIE): Means and Methods". Retrieved from buildingSMART Alliance: http://www.buildingsmartalliance.org/index.php/projects/cobie 